\title{
520 - Benefits of Assertive Community Treatment for Persons with Severe Mental Illness and Cancer
}

\author{
Monica Taylor-Desir
}

Breast cancer, the most commonly diagnosed cancer in women worldwide, is responsible for one in six cancer deaths (Sung, H. et al., 2021). Women with schizophrenia have an associated increased incidence of breast cancer compared to the general population (Grassi \& Riba, 2020). Patients with severe mental illness are noted to have disparities in accessing and initiating cancer treatment especially among those who are older (Iglay et al., 2017). A case vignette will be presented to illustrate the care and interventions provided by an American Assertive Community Treatment team which fostered supportive treatment engagement and improved the quality of life for a patient that chose to forgo recommended cancer treatment. This presentation will highlight the essential nature of the Assertive Community Treatment team in supporting decisional capacity, facilitation of a patient's grief and acknowledgement of one's own mortality as well as incorporation of medical and palliative care. The attendee will appreciate the importance of the multidisciplinary approach for persons with chronic mental illness and co-morbid cancer diagnoses.

Grassi, L., \& Riba, M. (2020). Cancer and severe mental illness: Bi-directional problems and potential solutions. Psycho-Oncology, 29(10), 1445-1451. https://doi.org/10.1002/pon.5534

Iglay, K., Santorelli, M. L., Hirshfield, K. M., Williams, J. M., Rhoads, G. G., Lin, Y., \& Demissie, K. (2017). Diagnosis and treatment delays among elderly breast cancer patients with pre-existing mental illness. Breast Cancer Research and Treatment, 166(1), 267-275. https://doi.org/10.1007/s10549017-4399-x

Sung, H., Ferlay, J., Siegel, R. L., Laversanne, M., Soerjomataram, I., Jemal, A., \& Bray, F. (2021). Global Cancer Statistics 2020: GLOBOCAN Estimates of Incidence and Mortality Worldwide for 36 Cancers in 185 Countries. CA: A Cancer Journal for Clinicians, 71(3), 209-249. https://doi.org/10.3322/caac.21660

\section{1 - The link between olfactory dysfunction and dementia: the road so far. Marcela Leão Petersen, Monia Bresolin, Ariane Madruga Monteiro}

Dementia is characterized by the presence of progressive cognitive impairment losses in the individual's social and occupational activities. Its etiological diagnosis has therapeutic and prognostic implications. Although its definitive diagnosis depends on neuropathological analysis, detailed anamnesis, physical and neuropsychological tests; biochemical and neuroimaging exams may enable a greater accuracy. Technological innovations using structural and functional neuroimaging methods, as well as biology and molecular genetics techniques, have presented perspectives for the early diagnosis of dementias, particularly Alzheimer's disease (AD). However, such techniques burden the diagnostic investigation, making its practice unfeasible most the times. The probable link between neurodegenerative diseases and impaired olfactory dysfunction has long been studied. It is suggested that smell tests can be used in dementia's early detection and differential diagnosis, reducing costs and facilitating the establishment of appropriate treatment. In order to verify the validity of this information, a medical literature search was carried out in may 2021 using PubMed and Cochrane Library, including the terms "olfaction" and "olfactory dysfunction" combined individually with "neurodegenerative disorder", "dementia" and "Alzheimer's disease". Only systematic reviews and meta-analyses written in English from 1991 to 2021 were included. Results show that olfactory impairment in neurodegenerative diseases worsens progressively as patients progress from mild cognitive impairment to AD. It suggests that odor tests could potentially identify $A D$ in the preclinical stages. Although, rigorously designed longitudinal cohort 